\title{
Mini-Open Approach for Direct Lateral Lumbar Interbody Fusion
}

\author{
Chong-Suh Lee ${ }^{1}$, Sung-Soo Chung ${ }^{1}$, Young-Ryeol Pae ${ }^{2}$, Se-Jun Park ${ }^{1}$ \\ ${ }^{1}$ Spine Center, Department of Orthopedic Surgery, Samsung Medical Center, Sungkyunkwan University School of Medicine, Seoul, Korea \\ ${ }^{2}$ Department of Orthopedic Surgery, Samsung Changwon Hospital, Sungkyunkwan University School of Medicine, Changwon, Korea
}

Study Design: Retrospective analysis.

Purposes: To introduce the mini-open lateral approach for the anterior lumbar interbody fusion (ALIF), and to investigate the advantages, technical pitfalls and complications by providing basic knowledge on extreme lateral interbody fusion (XLIF) or direct lumbar interbody fusion (DLIF).

Overview of Literature: Recently, minimally invasive lateral approach for the lumbar spine is revived and receiving popularity under the name of XLIF or DLIF by modification of mini-open method when using the sequential tubular dilator and special expandable retractor system.

Methods: Seventy-four patients who underwent surgery by the mini-open lateral approach from September 2000 to April 2008 with various disease entities were included. Blood losses, operation times, incision sizes, postoperative time to mobilization, length of hospital stays, technical problems and complications were all analyzed.

Results: The blood losses and operation times of patients who underwent simple ALIF were $61.2 \mathrm{~mL}$ and 86 minutes for one level, $107 \mathrm{~mL}$ and 106 minutes for two levels, $250 \mathrm{~mL}$ and 142.8 minutes for three levels, and $400 \mathrm{~mL}$ and 190 minutes for four levels of fusion. The incision sizes were on average $4.5 \mathrm{~cm}$ for one level, $6.3 \mathrm{~cm}$ for two levels, $8.5 \mathrm{~cm}$ for three levels and $10.0 \mathrm{~cm}$ for four levels of fusion. The complications were retroperitoneal hematoma (2 cases), pneumonia (1 case) and transient lumbosacral plexus palsy (3 cases).

Conclusions: Trials of mini-open lateral approach would be helpful before the trial of XLIF or DLIF. However, special attention is required for complications such as transient lumbosacral plexus palsy.

Keywords: Lumbar spine; Mini-open lateral approach; Interbody fusion

\section{Introduction}

There are various advantages of minimally invasive surgery, such as smaller incisions, minimal tissue dissection and injury, reduced blood losses, reduced hospital stays, quicker recovery and rehabilitation times, as well as improved cosmetic results. The current trend towards in- creased applications of minimally invasive surgery for all surgical fields in conjunction with patients' demands for such medical practices, are also likely to change the trend of spine surgery.

The minimally invasive operation in lumbar spine surgery has been drawing attention since Obenchain [1] first reported intervertebral discectomy and Mathews et al. [2]

Received May 23, 2013; Revised Jul 11, 2013; Accepted Jul 11, 2013

Corresponding author: Chong-Suh Lee

Department of Orthopedic Surgery, Samsung Medical Center, Sungkyunkwan University School of Medicine, 81 Irwon-ro, Gangnam-gu, Seoul 135-710, Korea

Tel: +82-2-3410-3509, Fax: +82-2-3410-0061, E-mail: csl@smc.samsung.co.kr 
reported intervertebral body fusion by laparoscopy. After the report of closed laparoscopic anterior lumbar interbody fusion [3,4], a mini-open lumbar interbody fusion technique was also introduced [3]. Nowadays, surgical incisions tend to be minimized with the mini-open approaches that are assisted by laparoscopy or microscopy, and sometimes with the naked eye. For mini-open anterior lumbar interbody fusions, the paramedian and anterolateral approaches which start at the lateral aspect of the rectus abdominis, are commonly used. Traditionally, lateral approaches to the lumbar spine through the psoas muscle have been used for the anterior fusions of lumbar spine, and the modifications of lateral approach with minimal incision have been reported. Recently, minimally invasive lateral approach is revived and gaining popularity under the name of the extreme lateral interbody fusion (XLIF) [5] or the direct lumbar interbody fusion (DLIF) $[6,7]$ through modifications of mini-open method using sequential tubular dilator, special expandable retractor system and intraoperative electromyography (EMG).

Because the mini-open lateral approach is commonly used for anterior lumbar interbody fusion at our center without intraoperative EMG, we investigated the advantages, disadvantages, and technical problems that should be considered when using this method.

\section{Materials and Methods}

\section{Patients}

Seventy-four patients who underwent surgery by the mini-open lateral approach from September 2000 to April 2008 formed the basis of this study. There were 33, $23,12,3$ cases with one, two, three, four levels fusion, 2 cases with curettage and bone graft, and 1 case of biopsy (Table 1). There were 36 men and 38 women with an average age of 44.1 years and 48.7 years, respectively. There were 2 cases of painful Schmorl's node, 11 cases of fractures, 10 cases of tuberculous spondylitis, 11 cases of pyogenic spondylitis, one case of fungal infections secondary to acute lymphocytic leukemia, 6 cases of internal disc derangements, 11 cases of degenerative lumbar kyphosis along with spinal stenosis, 7 cases of junctional kyphosis, 2 case of nonunions, one case of Charcot's spine, one case of postdiscectomy syndrome, 7 cases of idiopathic scoliosis, 3 cases of screw loosening and one case of lumbar Scheuermann's disease. The posterior approach was ad-
Table 1. Fusion levels and number of cases

Fusion level

Number

1 Level (33 cases)

$\begin{array}{lr}\text { T12-L1 } & 1 \\ \text { L1-L2 } & 4 \\ \text { L2-L3 } & 10 \\ \text { L3-L4 } & 11 \\ \text { L4-L5 } & 7\end{array}$

2 Levels (23 cases)

\begin{tabular}{ll} 
T12-L2 & 6 \\
L1-L3 & 4 \\
L2-L4 & 5 \\
L3-L5 & 6 \\
L1-L2, L4-L5 & 2 \\
3 Levels (12 cases) & \\
T12-L3 & 6 \\
L1-L4 & 2 \\
L2-L5 & 3 \\
T12-L1, L2-L4 & 1 \\
4 Levels (3 cases) & \\
L1-L5 & 2 \\
T12-L4 & 1 \\
\hline
\end{tabular}

ditionally used in 37 of these cases, excluding the internal disc derangements, painful Schmorl's node, infections, postdiscectomy syndromes, idiopathic scoliosis, lumbar Scheuermann's diseases, and one case of fracture. Among these 37 cases, anterior lumbar interbody fusion and posterior pedicle screw instrumentation was conducted on the same day for 25 cases, and the posterior pedicle screw instrumentation was performed 7 days after anterior lumbar interbody fusion in the others. Blood losses, operation times, incision sizes, time to standing, time to ambulation (walking more than 10 minutes), hospital stays, complications, technical problems, and the advantages and disadvantages of surgical methods were investigated in patients who underwent the mini-open lateral approach. For evaluation of blood losses and operation times, the patients were divided into two groups: 34 who underwent intervertebral lumbar interbody fusion after simple discectomy and fusion, and 37 who underwent additional manipulation of the vertebral body and soft tissues due to infection, fracture, or deformity (idiopathic scoliosis, Charcot's spine). The time to standing, time to 
ambulation and hospital stay were investigated only for those who underwent discectomy and one level anterior lumbar interbody fusion. Those who underwent more than two levels of fusion were excluded, as they were treated by the combined posterior approach due to deformities such as kyphosis, or due to systemic factors or delay in the ambulation period caused by infections or fractures. One-level cases in which posterior instrumentation was conducted were also excluded.

\section{Surgical techniques}

The patient was laid in true lateral position on the operating table. The table was bent at about $20^{\circ}$ of the waist level, and the hip and knee joints were flexed at about $30^{\circ}$. Under the guidance of a C-arm, an intervertebral disc and the anterior and posterior margin of the upper and lower adjacent vertebral bodies, which were to be fused, were being outlined. A line was drawn connecting the center of the upper and lower vertebral bodies to serve as guide for the incisions (Fig. 1). The abdominal muscles were split layer by layer in the direction of the muscle fibers, and after the posterior peritoneal fat pad had been retracted to the anterior side, the retroperitoneum was exposed and the psoas muscle, which covers the intervertebral disc, was split between anterior $1 / 3$ and posterior $2 / 3$ of psoas muscle in the direction of the muscle fibers exposed to the disc (Fig. 2). The anterior and posterior longitudinal ligaments were preserved, and the intervertebral disc was excised. An autogenous strut bone was harvested at the anterior iliac crest. After widening of intervertebral disc height with dilator, the cage or other types of bone graft can be inserted. If the height of the fusion gap was over $15 \mathrm{~mm}$, one mesh cage was inserted, and if the height was less than $14 \mathrm{~mm}$, one or two curved cages were utilized. In case of infections, only the autogenous iliac strut bone was inserted for fusions.

During approach, special self-retractor can be conveniently used. However, these special retractor are very expensive, and thus, if not available, the conventional type

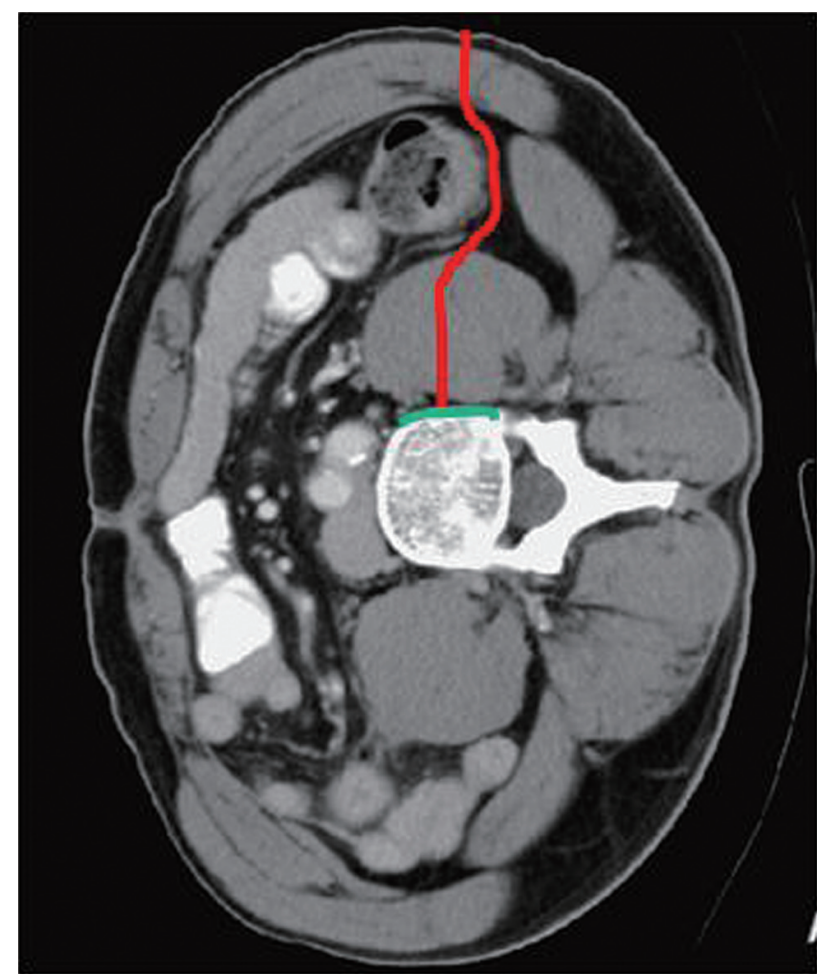

Fig. 2. Abdominopelvic computed tomography demonstrating the planes of mini-open lateral approach.
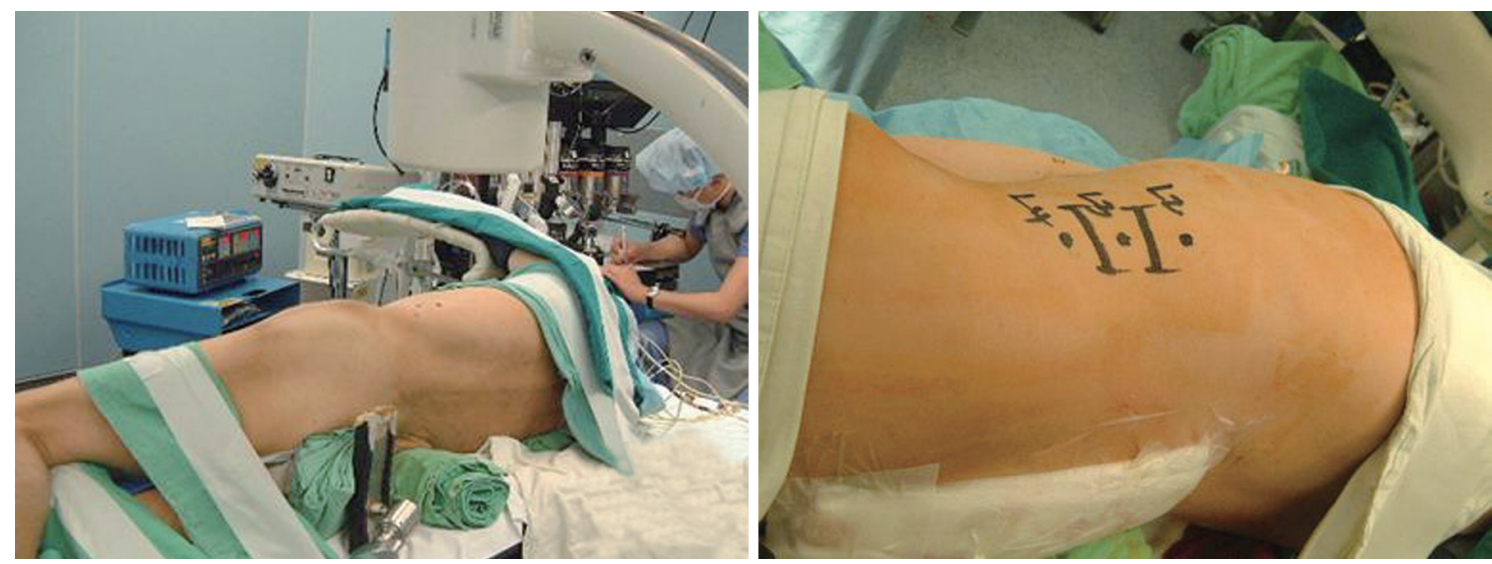

Fig. 1. Incision line between the center of the upper vertebral body and the center of the lower vertebral body under fluoroscopic guidance. 
of retractor can also be used.

For better illumination and magnified detailed vision, laparoscopy or microscopy can be used.

\section{Results}

\section{Blood loss and operation time}

The blood losses and operation times of the patients, who underwent discectomy and fusion without manipulation of the vertebral body and soft tissues, were $61.2 \mathrm{~mL}$ and 86 minute for one level (22 cases) and $107 \mathrm{~mL}$ and 106 minute for two levels (4 cases), $250 \mathrm{~mL}$ and 142.8 minute for three levels (7 cases), $400 \mathrm{~mL}$ and 190 minute for 4 levels ( 1 case), respectively (Table 2$)$. When debridement and corpectomy were additionally conducted due to infections or fractures, the blood losses and operation times showed increments (Table 3 ).

\section{Size of incision, time to standing and ambulation, and hospital stay}

The average length (range) of the surgical incision was $4.5 \mathrm{~cm}$ (range, $3.4-5.1 \mathrm{~cm}$ ) for one level, $6.4 \mathrm{~cm}$ (range, $5.1-7.2 \mathrm{~cm}$ ) for two levels, $8.5 \mathrm{~cm}$ (range, $7.0-9.4 \mathrm{~cm}$ ) for three levels, and $10.0 \mathrm{~cm}$ (range, $9.7-10.5 \mathrm{~cm}$ ) for four levels of fusion. The average time to standing and to ambulation were 1.7 days and 2.5 days, respectively, for patients who had no fractures or signs of infections and underwent only one level of anterior fusion without any posterior manipulations. The average hospital stay was 6.9 days.

\section{Complications}

Retroperitoneal hematoma occurred in two cases, pneumonia in one case, transient paralysis of the lumbosacral plexus in three cases, inadequate correction of kyphosis in one case, and the failure to insert lateral cages orthogonally to the disc space during anterior lumbar interbody fusion of the L4-5 level in one case. Retroperitoneal hematoma occurred in one patient who had acute lymphocytic leukemia with lumbar abscess and in one patient who underwent four levels of anterior and posterior lumbar fusions. Pneumonia occurred in a patient with active pulmonary tuberculosis. Transient lumbosacral plexus palsy occurred in three women who were over 60 years
Table 2. Blood losses and operation times in simple mini-open lateral ALIF

\begin{tabular}{lcc} 
Level (no. of patients) & EBL (mL) & $\begin{array}{c}\text { Operation time } \\
\text { (min) }\end{array}$ \\
\hline 1 Level (22) & 61.2 & 86.0 \\
\hline Levels (4) & 107.0 & 106.0 \\
\hline Levels (7) & 250.0 & 142.8 \\
\hline Levels (1) & 400.0 & 190.0 \\
\hline
\end{tabular}

ALIF, anterior lumbar interbody fusion; EBL, estimated blood loss.

Table 3. Blood losses and operation times in complex mini-open lateral ALIF (additional debridement and corpectomy)

\begin{tabular}{lrc} 
Level (no. of patients) & EBL (mL) & $\begin{array}{c}\text { Operation time } \\
\text { (min) }\end{array}$ \\
\hline 1 Level (14) & 281.8 & 165.7 \\
\hline L Levels (19) & 370.0 & 148.6 \\
\hline L Levels (5) & 528.0 & 197.0 \\
\hline L Levels (2) & $1,150.0$ & 220.0 \\
\hline
\end{tabular}

ALIF, anterior lumbar interbody fusion; EBL, estimated blood loss.

of age: two of these underwent a posterior operation in addition to one level and two levels of anterior lumbar interbody fusion, and the other underwent anterior lumbar interbody fusion alone of one level. Mild hip flexor weaknesses were found in all three patients, however, muscle powers recovered within 3 months. Since the L4-5 intervertebral disc was seated deeply into the iliac crests for one obese patient, an adequate exposure of the interbody disc was difficult, and insertion of only one lateral cage parallel to the disc space was not possible. Therefore, this patient underwent additional posterior instrumentation and fusion in considerations of postoperative instability or nonunion.

\section{Technical advantages and disadvantages}

With the mini-open lateral approach, access to the T12L1 vertebral body was possible without incising the diaphragm, and in one case of idiopathic scoliosis, the screw insertion at the T12 vertebral body was also possible. Moreover, with this approach, surgical treatment of various diseases, such as corrections of spinal deformities and corpectomy, was also feasible. However, for patients with kyphosis, the complete restoration of lumbar lordosis was sometimes difficult because it was not possible 
to achieve complete releases of anterior structures with small incisions. If the L4-5 intervertebral disc was deeply seated between the iliac crest, partial excisions of the iliac crest was required, and to approach the T12-L1 and L1-2 intervertebral discs, rib resections may be necessary.

\section{Discussion}

Ever since the laparoscopic anterior lumbar interbody fusion was introduced, the minimally invasive approach for anterior lumbar interbody fusion has been gaining interest $[3,8]$. Although laparoscopic anterior lumbar interbody fusion has advantages in terms of reduced blood losses, less pains, reduced admission and rehabilitation periods, and superior cosmetic effects, there are also some disadvantages [2,8-14]. In addition to the long learning curve, there are relatively high rates of occurrence for vascular, intestinal, and peritoneal injuries, ranging from $16.7 \%$ to $29.4 \%$. The mini-open lateral approach can be conducted without the aid of surgical microscopy or laparoscopy. However, it has the disadvantage of deep and narrow surgical field. Thus, only the operator can adequately visualize the region of interest, whereas other assistants of scrub nurses may not be aware of the progression on the operation due to poor visualization and lighting, and it may subsequently become difficult to conduct work which requires precision. Thus, by combining the mini-open approach with laparoscopy or microscopy has the advantage of securing a brighter and wider view on the region of interest. As a result, the assistants and scrub nurses can all visualize the operation, and thus, can actively participate in the operation, and the recording of the operation may also become easier. Also, when compared with closed laparoscopic surgery, even a surgeon who does not have experience with laparoscopic view can compare the monitor view with surgical fields through mini-open incisions, thus, gaining a chance to learn more easily.

However, if one is not dexterous with the use of laparoscopy, there is a disadvantage for time lost due to additional manipulation. Zdeblick and David [13] compared closed laparoscopic surgery with the mini-open approach for L4-5 anterior lumbar interbody fusion. They found that the rate of complications, such as vascular injury, was $20 \%$ for closed laparoscopic surgery, whereas, it was $4 \%$ for mini-open surgery. The rate of failure to insert two cages due to inadequate exposure was $16 \%$ for closed laparoscopic surgery, whereas, there were no failures to insert mini-open surgeries.

The mini-open lateral approach has several advantages when compared with the conventional mini-open approach through anterolateral or paramedian incisions. The other conventional mini-open approaches allow access to the L2-3 bodies proximally, whereas, the miniopen lateral approach allows a subdiaphragmatic approach to the T12-L1 bodies without incisions of the diaphragm. For accessing, there is no need to visualize the peritoneum or great vessels, and there is virtually no chance of damaging such structures. Moreover, because the anterior and posterior longitudinal ligaments are preserved, there is no risk of bone or cage displacements. Since the approach continues to go straight down from the lateral aspect, it is a very simple approach method as compared with others and is easier to learn. Since this technique approaches the side of the vertebral body, there is an advantage of anterior fixation of screws. However, through this method, the L5-S1 bodies cannot be approached, and if the L4-5 disc is seated deeply into iliac crests, the orthogonal access to the disc space is difficult. In our study, we also experienced difficulty to reach the L4-5 disc space, which resulted in unparallel placements of the cage in the direction of the disc space. Thus, in such cases, we partially excise the iliac crest approach. In the approach to the L1-2 and T12-L1 bodies, the 11th and 10th ribs may interfere. However, these ribs were not connected to the pleural cavity, thus, these ribs need to be cut and temporarily retracted upwards or downwards and reattached after the main procedures are completed.

The main disadvantage of this method is the need to split the psoas muscle in the mid-portion, thus, creating a risk of lumbosacral plexus injury. Three of our patients had such injuries, although all recovered within 3 months. Lumbosacral plexus is placed at posterior $1 / 2$ of vertebral bodies at L2 and L3 level. However, it is located at posterior half in L4 level. In L5 level, lumbosacral plexus is dispersed on the entire surface of lateral aspect in the vertebral body inside the posas muscle. Thus, we currently split the psoas muscle at the anterior one-third instead of the right middle portion. However, since transient lumbosacral plexus injury is also being reported in closed laparoscopic surgery through the retroperitoneum [8], it should not be considered as a complication limited to the lateral approach, even though the risk may be higher in the mini-open lateral approaches. 
This approach is most adequate for the operation for one or two levels of degenerative disc diseases with chronic low back pains or radiculopathy due to foraminal narrowing or internal disc derangement, like other miniopen approaches or closed laparoscopic surgeries $[11,13]$. However, we have employed the method more often for the corrections of burst fracture, infection, and kyphosis. Thus, this method could be utilized in such indications; however, in case of rigid kyphosis that require the release of anterior longitudinal ligament, an adequate correction may be difficult, and therefore, this method can not be recommended for rigid kyphosis.

In our case, for an attempt to correct one case of flat back syndrome, the adequate correction was not attained. In the correction of one case of idiopathic scoliosis, by making an incision of $10 \mathrm{~cm}$ and performing anterior instrumentation, a satisfactory result was gained, and thus, the application for a case of lumbar scoliosis was possible. The correction of thoraco-lumbar scoliosis can be performed through the anterior approach combined with thoracoscopy.

Regan et al. [11] conducted a multicenter comparison on the results of the laparoscopy-assisted mini-open approach and the closed laparoscopic approach, and found that mini-open surgery required much less operation time than closed laparoscopic surgery, but there was more bleeding and a slightly longer duration of hospital stay with this approach. The amount of blood loss with L4-5 interbody fusion was $232.3 \mathrm{~mL}$ and $134.4 \mathrm{~mL}$, the operation time was 147.9 minutes and 223.6 minutes, and the admission period was 4.1 days and 3.2 days, respectively. Zdeblick and David [13] reported that there were no significant differences in blood losses, operation times, and admission periods between the two groups; however, in closed laparoscopic surgery, the complication rate was four times higher. In general, blood losses and operation times were smaller in our study than in others, but the average admission period was longer. This may be due to the fact that the lateral approach involves no risk of major vessels during the approach, and such approach method is relatively simple. The longer admission period could be due to the different cultures of medical practice in different countries. The first ambulation was accomplished on an average of 2.5 days after surgery, and pain in the bone donor site was the major factor hindering ambulation.

Recently, minimally invasive lateral approach for the lumbar spine is revived and gaining popularity under the name of XLIF or DLIF. The XLIF and DLIF techniques are modifications of the retroperitoneal lateral approach to the lumbar spine $[5,6]$, and needs the same position and the same incision with mini-open lateral approach. XLIF needs one more incision for finger dissection of peritoneum, and to direct the guide pin, and to introduce the sequential dilator, and finally, act as a special retractor. Incision size is the same as mini-open lateral approach for one level fusion. The main difference between mini-open lateral interbody fusion and XLIF or DLIF is that the mini-open lateral interbody fusion is performed under the direct visualization, while XLIF or DLIF is the lack of direct visualization during approach. Therefore, there are higher risks of peritoneal, bowel, ureter injury and lumbosacral plexus injury which need neurophysiologic monitoring during the approach and are somewhat longer learning curves. The reported estimated blood loss in XLIF or DLIF for one or two level fusion and longer than two level fusion is $46 \mathrm{~mL}$ and $175 \mathrm{~mL}$, respectively [15]. Therefore, for the one or two level fusion, the miniopen approach is more appropriate than the XLIF or DLIF, because mini-open lateral approach is simpler and safer than XLIF or DLIF with very short learning curves and similar blood losses. For the long level fusion, the XLIF or DLIF would be more appropriate because the reported blood losses and operation times are much less [16]. Trials of the mini-open lateral approach would be helpful prior to the trials of XLIF or DLIF.

\section{Conclusions}

With the mini-open lateral approach for anterior lumbar interbody fusion, the relatively wide access to the T12L5 bodies is possible, and it is an effective method of approach which could be applied to various diseases. It is a safe operative technique, which involves practically no damages to the blood vessels, peritoneum, and intestines. It also has all the advantages of minimally invasive surgeries. Trials of mini-open lateral approach would be helpful before performing the XLIF or DLIF. However, one should be cautious for complications such as lumbosacral plexus paralysis.

\section{Conflict of Interest}

No potential conflict of interest relevant to this article was reported. 


\section{References}

1. Obenchain TG. Laparoscopic lumbar discectomy: case report. J Laparoendosc Surg 1991;1:145-9.

2. Mathews HH, Evans MT, Molligan HJ, Long BH. Laparoscopic discectomy with anterior lumbar interbody fusion. A preliminary review. Spine (Phila Pa 1976) $1995 ; 20: 1797-802$.

3. Mayer HM. A new microsurgical technique for minimally invasive anterior lumbar interbody fusion. Spine (Phila Pa 1976) 1997;22:691-9.

4. Dewald CJ, Millikan KW, Hammerberg KW, Doolas A, Dewald RL. An open, minimally invasive approach to the lumbar spine. Am Surg 1999;65:61-8.

5. Ozgur BM, Aryan HE, Pimenta L, Taylor WR. Extreme Lateral Interbody Fusion (XLIF): a novel surgical technique for anterior lumbar interbody fusion. Spine J 2006;6:435-43.

6. Knight RQ, Schwaegler P, Hanscom D, Roh J. Direct lateral lumbar interbody fusion for degenerative conditions: early complication profile. J Spinal Disord Tech 2009;22:34-7.

7. Bergey DL, Villavicencio AT, Goldstein T, Regan JJ. Endoscopic lateral transpsoas approach to the lumbar spine. Spine (Phila Pa 1976) 2004;29:1681-8.

8. McAfee PC, Regan JJ, Geis WP, Fedder IL. Minimally invasive anterior retroperitoneal approach to the lumbar spine. Emphasis on the lateral BAK. Spine (Phila Pa 1976) 1998;23:1476-84.

9. Levrant SG, Bieber EJ, Barnes RB. Anterior abdominal wall adhesions after laparotomy or laparoscopy. J Am Assoc Gynecol Laparosc 1997;4:353-6.
10. McAfee PC, Regan JR, Zdeblick T, et al. The incidence of complications in endoscopic anterior thoracolumbar spinal reconstructive surgery. A prospective multicenter study comprising the first 100 consecutive cases. Spine (Phila Pa 1976) 1995; 20:1624-32.

11. Regan JJ, Yuan H, McAfee PC. Laparoscopic fusion of the lumbar spine: minimally invasive spine surgery. A prospective multicenter study evaluating open and laparoscopic lumbar fusion. Spine (Phila Pa 1976) 1999;24:402-11.

12. Tiusanen H, Seitsalo S, Osterman K, Soini J. Retrograde ejaculation after anterior interbody lumbar fusion. Eur Spine J 1995;4:339-42.

13. Zdeblick TA, David SM. A prospective comparison of surgical approach for anterior L4-L5 fusion: laparoscopic versus mini anterior lumbar interbody fusion. Spine (Phila Pa 1976) 2000;25:2682-7.

14. Regan JJ, McAfee PC, Mark MJ. Atlas of endoscopic spine surgery. St. Louis, MO: Quality Medical Publishing; 1995.

15. Karikari IO, Grossi PM, Nimjee SM, et al. Minimally invasive lumbar interbody fusion in patients older than 70 years of age: analysis of peri- and postoperative complications. Neurosurgery 2011;68:897-902.

16. Isaacs RE, Hyde J, Goodrich JA, Rodgers WB, Phillips FM. A prospective, nonrandomized, multicenter evaluation of extreme lateral interbody fusion for the treatment of adult degenerative scoliosis: perioperative outcomes and complications. Spine (Phila Pa 1976) 2010;35(26 Suppl):S322-30. 